\title{
Estudo da prevalência de sangramento uterino anormal na Amazônia Ocidental: aspectos clínicos e epidemiológicos
}

\author{
Study of the prevalence of abnormal uterine bleeding in the Western Amazon: clinical and \\ epidemiological aspects
Estudio de la prevalecía de sangramiento uterino anormal en la Amazonia Occidental: aspectos clínicos y epidemiológicos

Gabriela Bentes de Sousa ${ }^{1 *}$, Aldenícia Fernanda Saraiva dos Santos ${ }^{1}$, Thiago Gester Affonso ${ }^{1}$, Thaís Cavazzani Trombetta ${ }^{2}$, Leilane Bentes de Sousa ${ }^{3}$, Dária Barroso Serrão das Neves¹.

\section{RESUMO}

Objetivos: avaliar o perfil clínico, epidemiológico e tratamentos instituídos às pacientes com Sangramento Uterino Anormal (SUA) atendidas em ambulatório de Ginecologia Endócrina, em Manaus-Amazonas, de Julho de 2015 a Julho de 2017. Métodos: Trata-se de um estudo retrospectivo, baseado na análise de prontuários médicos arquivados. Os dados foram coletados através de uma ficha de coleta de dados, tabulados e analisados no SPSS e organizados em tabelas e gráficos no Excel. Resultados: Foram atendidas 909 pacientes, destas 201 por SUA (22,1\%). A maioria possuía mais de 35 anos $(72,6 \%)$, relatou fluxo menstrual aumentado (91\%) associado a dismenorreia $(66,2 \%)$. Quanto as etiologias, segundo PALM - COEIN, a mais prevalente das estruturais foi leiomiomatose $(65,7 \%)$ e das não estruturais endometrial $(13,4 \%)$. O tratamento foi cirúrgico em $70,6 \%$ dos casos. Em $69,7 \%$ negaram tratamento prévio e a média do tempo de evolução da doença foi de 27 meses. Conclusões: A prevalência de SUA foi maior que a encontrada em países subdesenvolvidos. A leiomiomatose foi a causa mais comum entre todas as causas. $O$ tipo de tratamento em maior frequência foi cirúrgico. Chamou atenção os casos de abandono do tratamento e o tempo de evolução da doença, comprometendo a qualidade de vida e saúde destas pacientes.

Palavras-chave: Hemorragia uterina, Saúde da mulher, Leiomiomatose.

\begin{abstract}
Objectives: To evaluate the clinical and epidemiological profile and treatments instituted to patients with Abnormal Uterine Bleeding (AUB) attended at the Endocrine Gynecology outpatient clinic in ManausAmazonas, from July 2015 to July 2017. Methods: This is a retrospective study, based on the analysis of archived medical records. The data were collected through a datasheet, tabulated and analyzed in SPSS and organized into tables and graphs in Excel. Results: A total of 909 patients were attended, of these 201 by AUB $(22.1 \%)$. The majority were older than 35 years $(72.6 \%)$, reported increased menstrual flow $(91 \%)$ associated with dysmenorrhea (66.2\%). Regarding the etiologies, according to PALM - COEIN, the most prevalent of the structural was leiomyomatosis $(65.7 \%)$ and non - structural endometrial $(13.4 \%)$. The treatment was surgical in $70.6 \%$ of the cases. In $69.7 \%$ they denied previous treatment and the mean time of disease evolution was 27 months. Conclusions: The prevalence of AUB was higher than that found in underdeveloped countries. Leiomyomatosis was the most common cause among all causes. The most frequent type of treatment was surgical. Called attention the cases of treatment abandonment and disease progression, compromising the quality of life and health of these patients.
\end{abstract}

Key words: Uterine hemorrhage, Women's health, Leiomyomatosis.

1 Universidade do Estado do Amazonas (UEA), Manaus-AM. *E-mail: gabriela.bentes.sousa@gmail.com

2 Universidade Nilton Lins (UNL), Manaus-AM.

${ }^{3}$ Universidade Federal do Amazonas (UFAM), Manaus-AM.

SUBMETIDO EM: 7/2019

ACEITO EM: $8 / 2019$

PUBLICADO EM: 10/2019 


\section{RESUMEN}

Objetivos: Evaluar el perfil clínico, epidemiológico y tratamientos dados a los pacientes con Sangramientos Uterinos Anormales (SUA) atendidas en centros de salud de Ginecología Endocrinóloga, en ManaosAmazonas, de julio de 2015 a Julio 2017. Métodos: Se trata de un estudio retrospectivo, que se basa en el análisis de historias clínicas archivadas. Los datos fueron recolectados a través de una ficha de colecta de datos, tabulados y analizados em SPSS y organizados en tablas y gráficos en Excel. Resultados: fueron atendidos 909 pacientes, de estas 201 por SUA (22,1\%). La mayoría tenían más de 35 años (72,6\%), con aumento del flujo menstrual (91\%) asociado con dismenorrea (66,2\%). Con respectos a las etiologías, según PALM-COEIN, el más prevalente de las estructurales fue leiomiomatosis $(65,7 \%)$ y de las no estructurales endometrial (13,4\%). El tratamiento fue quirúrgico en $70,6 \%$ de los casos. En $69,7 \%$ negaron tratamiento previo y el tiempo promedio de evolución de la enfermedad fue de 27 meses. Conclusiones: la prevalencia de SUA fue mayor que la encontrada en países subdesarrollados. A leiomiomatosis fue la causa más común entre las causas. El tipo de tratamiento en mayor frecuencia fue quirúrgico. Lo que llamó la atención fueron los casos de abandono de tratamiento y el tiempo de evolución de la enfermedad, comprendiendo la calidad de vida y salud de estos pacientes.

Palabras claves: Hemorragia uterina, Salud de la mujer, Leiomiomatosis.

\section{INTRODUÇÃO}

O Sangramento Uterino Anormal (SUA) é definido como perda menstrual excessiva podendo ocorrer isoladamente ou em combinação com outros sintomas e com uma perda menstrual menor que $80 \mathrm{~mL}$. Esta definição tornou-se útil, para as mulheres que não preenchiam o antigo critério padrão de perda menstrual acima de $80 \mathrm{~mL}$ para classificar como sangramento menstrual excessivo, sendo esta perda sanguínea, apesar do volume, fator que interferia negativamente na sua qualidade de vida e cotidiano (NATIONAL COLLABORATING CENTRE FOR WOMEN'S AND CHILDREN'S HEALTH, 2007; SRIPRASERT I et al., 2017)

O SUA acomete até $40 \%$ das mulheres no mundo impactando negativamente na qualidade de vida das mesmas, as quais tem seus relacionamentos e vida social prejudicados em $2 / 3$ dos casos (YELA DA, BENETTI-PINTO CL, 2018). É causa de um terço de todas as consultas ginecológicas de mulheres na prémenopausa e $70 \%$ das mulheres na perimenopausa e pós menopausa sendo responsável também por altos custos econômicos. O custo direto anual com pacientes com SUA em 2007 foi de aproximadamente US\$ 1 bilhão e indireto de aproximadamente US\$ 12 bilhões (MATTHEWS ML, 2015). Nos países em desenvolvimento, o SUA acomete 5-15\% das pacientes em idade reprodutiva e uma porcentagem maior em mulheres com idade avançada (HARLOW SD e CAMPBELL OMR, 2004).

Em 2011, a Federação Internacional de Ginecologia e Obstetrícia, propôs uma revisão da nomenclatura utilizada para as alterações relacionadas ao ciclo menstrual e a classificação das etiologias para o SUA utilizando o acrônimo PALM - COEIN (P-Pólipo, A-Adenomiose, L-Leiomiomatose, MMalignidade/hiperplasia - C- Coagulopatia, O - Distúrbios ovarianos, E- Endometrial, I- latrogênica, N- Não classificada em outra parte), com o objetivo de facilitar o diagnóstico e manejo das condutas relacionadas ao SUA. Assim, termos antes utilizados para os distúrbios menstruais foram substituídos por Sangramento Uterino Anormal e as etiologias estratificadas em nove categorias, sendo as estruturais: Pólipo (SUA - P), Adenomiose (SUA - A), Leiomiomatose (SUA - L), Malignidade e/ou hiperplasia (SUA - M) e as não estruturais: Coagulopatias (SUA - C), disfunção ovulatória (SUA - O), Endometrial (SUA - E), latrogênica (SUA - I) e as não classificadas em outra parte (SUA- N) (MUNRO MG et al., 2011).

O tratamento de SUA pode ser medicamentoso hormonal e/ou não hormonal e/ou cirúrgico quando há falha do tratamento clínico ou contraindicações ou não foi aceito pela paciente. Dentre as opções não hormonais incluem-se AINES (Anti-inflamatórios Não Esteroidais) e o ácido tranexâmico. Das hormonais incluem-se: anticoncepcionais orais combinados, progestágenos em suas diversas formas de apresentação, 
análogos do GnRH (Hormônio Liberador de Gonadotrofina) e acetato de ulipristal. Das opções cirúrgicas quando há desejo de preservação da fertilidade e presença de miomas pequenos, as opções são ablação endometrial, miomectomia, polipectomia e embolização das artérias uterinas. Quando não há desejo de engravidar e manutenção do útero, a histerectomia é a escolha (FILHO ALS et al., 2015).

Considerando-se a carência por estudos que abordem a temática na cidade de Manaus-Amazonas e a observação cada vez mais frequente de atendimentos por SUA em unidade secundária de saúde na capital Amazonense, bem como serviço de urgência ginecológica, o impacto do ponto de vista de saúde, social e psicológico que as pacientes acometidas por SUA sofrem, além da ausência de padronização do atendimento e divergência de condutas na prática clínica, o objetivo deste trabalho foi avaliar o perfil clínico, epidemiológico e tratamentos instituídos às pacientes com sangramento uterino anormal atendidas no ambulatório de Ginecologia Endócrina, em unidade de atendimento secundário de saúde na cidade de Manaus-AM, no período de julho de 2015 a julho de 2017.

\section{MÉTODOS}

Estudo retrospectivo baseado em dados obtidos por meio de prontuários médicos arquivados no serviço de arquivos médicos de Manaus - AM. A população de estudo abrangeu as pacientes atendidas, por sangramento uterino anormal, no ambulatório de Ginecologia Endócrina da Policlínica PAM Codajás, no período de julho de 2015 a julho de 2017. Por não haver registros na literatura da estatística local de atendimentos por SUA, foi considerada toda a demanda de atendimentos por esta causa nesse período neste ambulatório.

Foram incluídos na pesquisa as pacientes atendidas por sangramento uterino anormal no período de julho de 2015 a julho de 2017, no ambulatório de Ginecologia Endócrina, e excluídos os casos os prontuários cujo preenchimento foi prejudicado e/ou danificados pelas condições ambientais, que estejam ilegíveis ou que não estiverem preenchidos.

A análise dos dados compreendeu as seguintes etapas: identificação do perfil epidemiológico das pacientes atendidas por SUA que tenham ocorridos na unidade de atendimento secundário no período julho de 2015 a julho de 2017, identificação do perfil clínico e causas de SUA e identificação dos principais tratamentos instituídos nas pacientes atendidas por SUA. Os dados foram coletados por meio de uma ficha de coleta de dados.

Os dados foram apresentados por meio de gráficos e tabelas, onde se calculou as frequências absolutas simples e relativas para os dados categóricos. $\mathrm{Na}$ análise dos dados quantitativos, quando aceita a hipótese de normalidade por meio do teste de Shapiro-Wilk, foi calculada a média e o desvio-padrão (Dp). No entanto, quando rejeitada a hipótese de normalidade, foi calculada a mediana. Já na análise dos dados categóricos foi aplicado o teste do qui-quadrado de Pearson, sendo que na impossibilidade de aplicar o teste de Pearson, foi aplicado o teste exato de Fisher para as tabelas 2x2. Na comparação das médias foi aplicado o teste t-student ou ANOVA para os dados paramétricos e para comparação das medianas os testes não-paramétricos de Mann-Whitney ou Kruskal-Wallis, o nível de significância fixado nos testes estatísticos foi de 5\% (VIEIRA S, 2004).

Este projeto foi submetido ao Comitê de Ética em Pesquisa envolvendo seres humanos da Universidade do Estado do Amazonas segundo CAAE 69067417.6.0000.5016, conforme parecer número 2.227.112, emitido em 18 de agosto de 2017.

\section{RESULTADOS}

No período de julho de 2015 a julho de 2017 foram atendidas 909 pacientes nos ambulatórios de ginecologia geral. Destes, 201 casos eram de Sangramento Uterino Anormal, o que corresponde a 22,1\% do total de atendimentos. Quanto ao perfil epidemiológico a maioria das pacientes atendidas com SUA pertenciam a faixa etária acima de 35 anos $(72,6 \%)$, possuíam $2^{\circ}$ grau de escolaridade $(31,3 \%)$, eram da cor parda $(62,7 \%)$, estado civil casada (39,8\%) e natural de Manaus (53,7\%). A média de idade das pacientes com SUA foi de 41,6 anos. Sendo a menor idade de 17 anos e a maior idade de 74 anos (Tabela 1). 
Tabela 1 - Perfil epidemiológico das pacientes com Sangramento Uterino Anormal atendidas no ambulatório de ginecologia da Policlínica PAM Codajás de julho de 2015 a julho de 2017. Variável Frequência absoluta simples $\%$

\begin{tabular}{|c|c|c|}
\hline \multicolumn{3}{|l|}{ Faixa etária } \\
\hline \multicolumn{3}{|l|}{$<15$ anos } \\
\hline 15-35 anos & 54 & 27 \\
\hline$>35$ anos & 146 & 72,6 \\
\hline Sem informação & 1 & 0,5 \\
\hline Total & 201 & 100 \\
\hline \multicolumn{3}{|l|}{ Escolaridade } \\
\hline Nenhuma & 5 & 2,5 \\
\hline $1^{\circ}$ grau & 56 & 27,9 \\
\hline $2^{\circ}$ grau & 63 & 31,3 \\
\hline Ensino superior & 39 & 19,4 \\
\hline Sem preenchimento & 38 & 18,9 \\
\hline Total & 201 & 100 \\
\hline \multicolumn{3}{|l|}{ Cor/raça } \\
\hline Branca & 20 & 10 \\
\hline Negra & 18 & 9 \\
\hline Parda & 126 & 62,7 \\
\hline Sem preenchimento & 37 & 18,4 \\
\hline Total & 201 & 100 \\
\hline \multicolumn{3}{|l|}{ Estado civil } \\
\hline Casada & 80 & 39,8 \\
\hline Solteira & 76 & 37,8 \\
\hline Divorciada & 6 & 3 \\
\hline Viúva & 5 & 2,5 \\
\hline Outro & 26 & 12,9 \\
\hline Sem preenchimento & 8 & 4 \\
\hline Total & 201 & 100 \\
\hline \multicolumn{3}{|l|}{ Naturalidade } \\
\hline Manaus & 108 & 53,7 \\
\hline Interior do Amazonas & 32 & 15,9 \\
\hline Outros estados & 31 & 15,4 \\
\hline Sem preenchimento & 30 & 14,9 \\
\hline Total & 201 & 100 \\
\hline
\end{tabular}

Fonte: Sousa GB, Santos AFS, Affonso TG, 2019.

O diagnóstico de SUA foi baseado na história clínica, exames laboratoriais quando se fizeram necessários dentre os principais: hemograma, dosagens de hormônios tireoidianos e prolactina e/ou exames de imagem como a ultrassonografia e ressonância nuclear magnética. Os exames laboratoriais foram solicitados em 
$58,7 \%$, exames de imagem foram utilizados na propedêutica complementar em $92 \%$ dos casos e o exame de imagem mais frequente foi a ultrassonografia transvaginal $(85,6 \%)$, seguido da ressonância nuclear magnética de pelve $(19,5 \%)$.

$\mathrm{Na}$ história clínica ao serem questionadas na consulta sobre seu padrão de sangramento menstrual as mesmas consideraram com maior frequência ter fluxo menstrual regular $(62,7 \%)$ e aumentado (91\%) com presença de dismenorreia moderada a intensa $(66,2 \%)$. Os dados do exame físico das pacientes foram incompletos ou não descritos no prontuário porém relatos como: presença de fundo uterino palpável acima da cicatriz umbilical, dor a palpação do abdome, dor a mobilização do colo e palpação dos anexos, abaulamento em fundo de saco, abaulamento em anexos, presença de conteúdo sanguinolento no canal vaginal, presença de ectopia cervical, colo endurecido à palpação, presença de pólipo cervical e leucorreia foram os mais frequentes.

Outros dados da história ginecológica destacam-se, a maior porcentagem encontrada foi multípara $(50,7 \%)$. Ao serem questionadas na consulta sobre seu padrão de sangramento menstrual referiram fluxo menstrual aumentado (91\%), apresentavam dismenorreia em $66,2 \%$ dos casos variando de moderada a intensa, utilizavam algum método contraceptivo $(50,7 \%)$ seja cirúrgico por meio de laqueadura tubária ou preservativo e/ou contraceptivo oral ou injetável e não possuíam histórico de neoplasia ginecológica na família $(87,1 \%)$ e negaram infecções sexualmente transmissíveis $(51,7 \%)$.

A média do tempo de evolução da doença em meses foi de 27 meses, ou seja, a média de tempo em que a paciente começou a apresentar sintomatologia de Sangramento Uterino Anormal até a procura de atendimento no serviço de ginecologia, foi de aproximadamente 2 anos e 3 meses. Quando relacionada a mediana do tempo de evolução da doença e o tipo de tratamento realizado, observou-se que as pacientes que evoluíam há mais tempo com SUA referiram histórico de tratamento clínico, compreendendo tratamento hormonal e/ou não hormonal e posteriormente evoluíram para tratamento cirúrgico $(p<0,05)$. Houve relevância estatística nos dados apresentados (Gráfico 1).

Gráfico 1 - Comparação das medianas do tempo de evolução da doença em relação ao tipo de tratamento das mulheres amostradas, Manaus - AM.

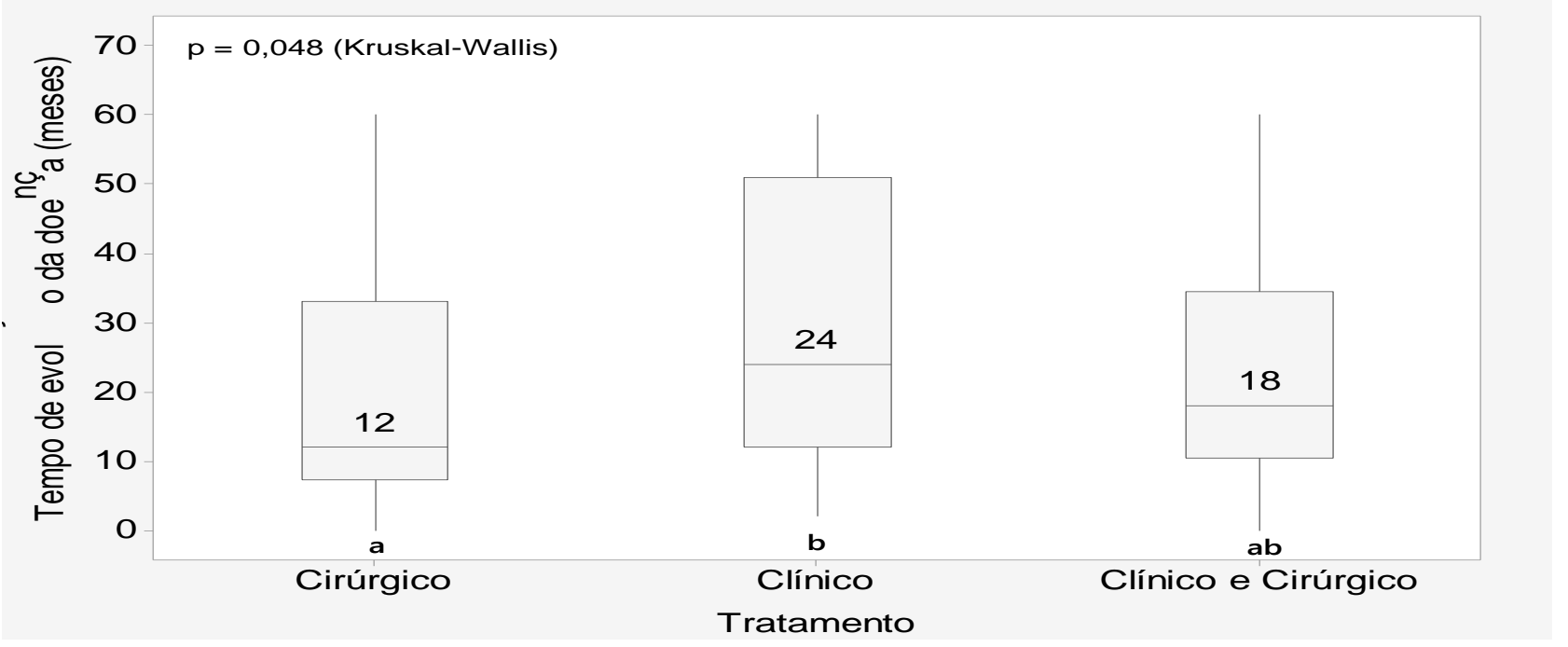

Fonte: Sousa GB, Santos AFS, Affonso TG, 2019.

Quando comparada a mediana do tempo de evolução da doença em relação a presença ou ausência de tratamento prévio, observou-se que as pacientes que evoluíam há mais tempo com SUA possuíam história de tratamento prévio $(p=0,427)$ porém não houve significância estatística pelo teste estatístico aplicado (Gráfico 2). 
Gráfico 2 - Comparação das medianas do tempo de evolução da doença em relação ao tratamento prévio por parte das mulheres amostradas, Manaus - AM.

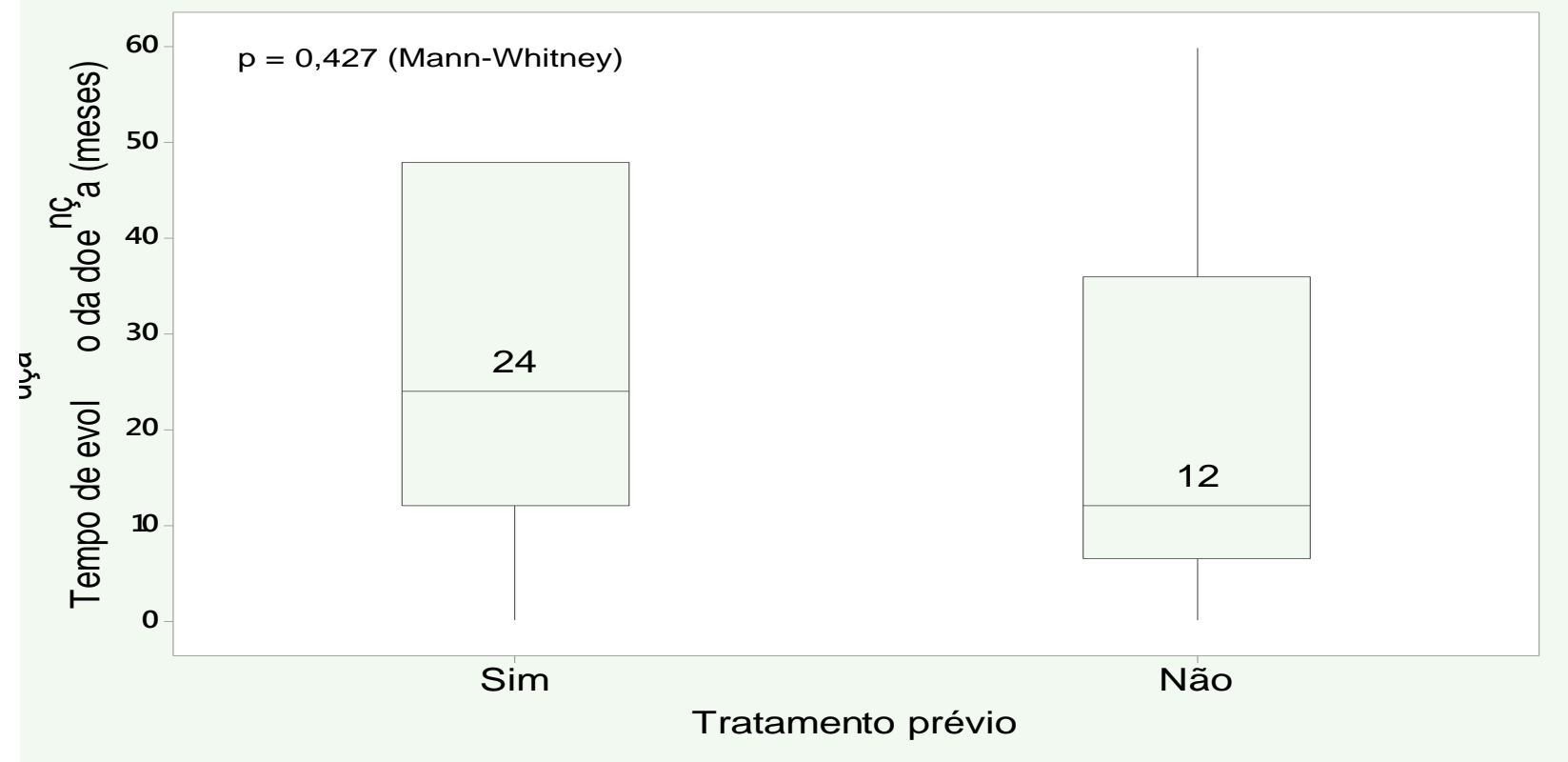

Fonte: Sousa GB, Santos AFS, Affonso TG, 2019.

O tratamento dos casos de SUA foi cirúrgico em $70,6 \%$ dos casos e a histerectomia foi o procedimento de escolha em $35,3 \%$, as demais pacientes foram seguidas clinicamente com análogo de GnRh $(24,9 \%)$, uma $(11,9 \%)$, duas $(8 \%)$ ou três doses $(2 \%)$, seguido de contraceptivo combinado $(17,9 \%)$, progestágeno (10\%) e anti-inflamatório não hormonal (10,4\%). Houve abandono de tratamento em $37,3 \%$ dos casos e quando questionada sobre histórico de tratamento prévio, as mesmas negaram em 69,7\% dos casos (Tabela 2). Em relação ao número de doses de análogo de $\mathrm{GnRH}$ realizadas nas pacientes submetidas ou não a tratamento cirúrgico, observou-se que a média do número de doses de análogo de $\mathrm{GnRH}$ foi de 3,46 nas submetidas a tratamento cirúrgico e 3,39 nas que não foram submetidas a tratamento cirúrgico. Quando essas médias foram comparadas, não houve relevância estatística $(p=0,724)$, conforme mostra o Gráfico 3.

Gráfico 3 - Comparação das médias do número de doses de análogo de GnRH em relação ao tratamento cirúrgico das mulheres amostradas, Manaus - AM.

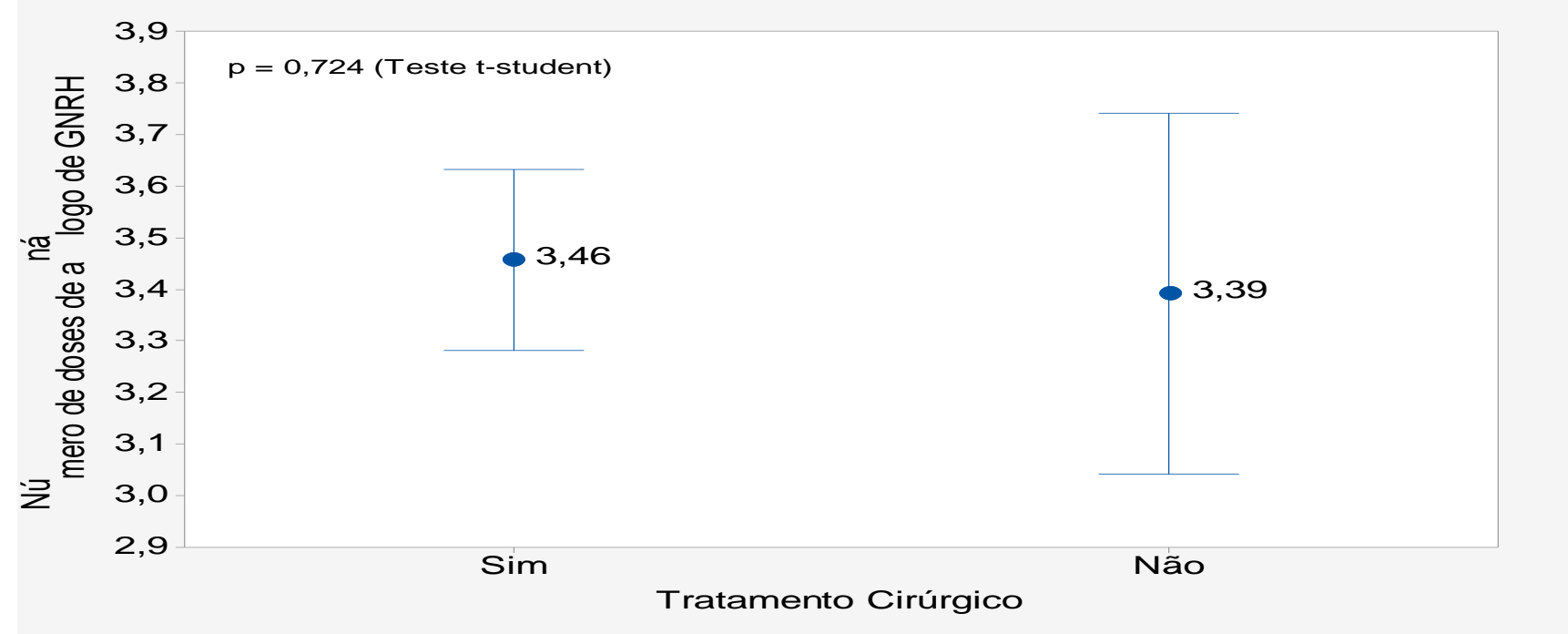

Fonte: Sousa GB, Santos AFS, Affonso TG, 2019. 
Quanto as etiologias do Sangramento Uterino Anormal, das causas estruturais a mais frequente foi Leiomiomatose uterina $(65,7 \%)$, seguida de pólipo endometrial $(21,9 \%)$, Adenomiose $(8,5 \%)$ e malignidade/hiperplasia (4,5\%). Em se tratando das patologias não estruturais a mais prevalente foi a causa endometrial $(13,4 \%)$, seguida da não classificada em outra parte $(11,4 \%)$, disfunção ovulatória $(2,5 \%)$, coagulopatia $(1,5 \%)$, dados mostrados na Tabela 2.

Tabela 2 - Etiologias de Sangramento Uterino Anormal, segundo a classificação PALMCOEIN das pacientes atendidas no ambulatório de ginecologia da Policlínica PAM Codajás de julho de 2015 a julho de 2017.

\begin{tabular}{lcc}
\hline \multicolumn{1}{c}{ Etiologias de SUA segundo } & $\begin{array}{c}\text { Frequência absoluta } \\
\text { simples }\end{array}$ & $\%$ \\
\hline Pólipo & 44 & 21,9 \\
Adenomiose & 17 & 8,5 \\
Leiomiomatose & 132 & 65,7 \\
Malignidade/hiperplasia & 9 & 4,5 \\
Coagulopatia & 3 & 1,5 \\
Disfunção Ovulatória & 5 & 2,5 \\
Endometrial & 27 & 13,4 \\
latrogênica & 0 & 0 \\
Não classificada em outra parte & 23 & 11,4 \\
\hline
\end{tabular}

Fonte: Sousa GB, Santos AFS, Affonso TG, 2019.

Em relação a classificação da miomatose uterina, o tipo intramural $(47,3 \%)$ destacou-se entre os tipos mais frequentes, seguido do submucoso $(18,9 \%)$ e subseroso $(15,9 \%)$. Vale destacar que muitas pacientes não possuíam apenas um tipo de mioma e que as análises foram feitas de forma independente. Das pacientes com mioma submucoso, o tipo de tratamento foi cirúrgico em $84,2 \%$ dos casos.

Quando o tratamento foi cirúrgico, a frequência das patologias estruturais confirmadas por exame histopatológico foi: leiomiomatose uterina $(23,4 \%)$, pólipo endometrial $(14,4 \%)$, não classificada em outra parte $(6 \%)$ e malignidade/hiperplasia e adenomiose (4,5\%), conforme mostrado na Tabela 3.

Tabela 3 - Diagnóstico por exame histopatológico das pacientes com Sangramento Uterino Anormal atendidas no ambulatório de ginecologia da Policlínica PAM Codajás de julho de 2015 a julho de 2017.

\begin{tabular}{lcc}
\hline $\begin{array}{c}\text { Etiologias estruturais confirmadas por } \\
\text { histopatológico }\end{array}$ & $\begin{array}{c}\text { Frequência absoluta } \\
\text { simples }\end{array}$ & $\%$ \\
\hline Pólipo & 29 & 14,4 \\
Adenomiose & 9 & 4,5 \\
Leiomiomatose & 47 & 23,4 \\
Malignidade/hiperplasia & 9 & 4,5 \\
Não classificada em outra parte & 12 & 6 \\
\hline
\end{tabular}

Fonte: Sousa GB, Santos AFS, Affonso TG, 2019.

\section{DISCUSSÃO}

Os resultados encontrados acerca da prevalência de 22\% de casos SUA está acima da prevalência para países subdesenvolvidos de 5 a 15\% (HARLOW SD e CAMPBELL OMR, 2004), mas ainda dentro da prevalência mundial de 4 a 27\% (MONTEIRO IU, 2015). As características epidemiológicas da maioria das pacientes atendidas por SUA como faixa etária acima de 35 anos, juntamente com o perfil clínico ginecológico de fluxo menstrual aumentado e relevante índice de dismenorreia demonstram o quanto é uma condição comum e, às vezes, debilitante em mulheres em idade reprodutiva. Essa condição pode comprometer suas atividades da vida diária, relacionamentos e produtividade no mercado de trabalho (SINGH S et al., 2013). 
Estudos apontam que o SUA é a causa direta de uma carga significativa de cuidados de saúde para as mulheres, suas famílias e a sociedade como um todo. Até 30\% das mulheres procurarão assistência médica para esse problema durante seus anos reprodutivos (FILHO ALS et al., 2016; MATTHEWS ML, 2015; SINGH $S$ et al., 2018; YELA DA, BENETTI-PINTO CL, 2018).

Considerando-se as demais características epidemiológicas a maior prevalência de SUA na raça parda dentre as pacientes atendidas no serviço secundário de saúde de Manaus, deve-se a maior prevalência da raça parda no Amazonas (INSTITUTO BRASILEIRO DE GEOGRAFIA E ESTATÍSTICA, 2011).

Dentre os fatores socioeconômicos, a escolaridade é importante e essencial para adesão aos tratamentos propostos, visto que a baixa adesão é maior naqueles indivíduos com menor escolaridade (TAVARES NUL et al., 2016). Em nosso estudo a maior frequência de SUA foi nas pacientes que possuíam segundo grau, o que é fator positivo para entendimento do binômio saúde-doença, compreensão da condição de saúde pela qual estão acometidas e seus tratamentos.

O diagnóstico de SUA foi clínico e baseado em exames de imagem como ultrassonografia transvaginal e em alguns casos ressonância nuclear magnética de pelve. Os sintomas mais frequentemente relatados foram aumento do fluxo menstrual e dismenorreia moderada a intensa. Dados do exame físico como útero aumentado de volume, com fundo uterino palpável à nível abdominal e abaulamento em regiões de anexos foram descritos no exame físico. Esses dados corroboram com o protocolo do National Institute for Health and Care Excellence (NICE) o qual recomenda que a propedêutica complementar deve ser solicitada conforme dados da história clínica e exame físico, nos acasos em que o útero está palpável a nível abdominal, evidência de massa pélvica de origem incerta e também na falha do tratamento clínico. A ultrassonografia pélvica/transvaginal deve ser a primeira linha de investigação de patologia estrutural nos casos de SUA (NATIONAL INSTITUTE FOR HEALTH AND CARE EXCELLENCE, 2018).

Não foram encontrados estudos no Amazonas e Brasil em relação a prevalência de SUA e suas etiologias. Os resultados do nosso trabalho corroboram com estudo realizado na Índia em que a etiologia mais frequente foi Leiomiomatose tanto pelo diagnóstico clínico (41,1\%) quanto histopatológico (37,2\%) (MISHRA D e SULTAN S, 2017).

A Leiomiomatose e Adenomiose também foram estudadas em relação a sua prevalência em peças de histerectomia recebidas em hospital de Kumaon, Índia, e a maior prevalência foi de Adenomiose (46,34\%) (RIZVI G et al., 2013).

Em estudo realizado na China sobre prevalência de SUA segundo etiologia PALM-COEIN a etiologia mais frequente foi disfunção ovulatória (57,7\%) (SUN Y et al., 2018). Em se tratando do tipo de mioma, o mais prevalente em nosso estudo foi o Intramural seguido do Submucoso, achado diferente do encontrado em pesquisa realizada na Espanha, para avaliar o perfil clínico e epidemiológico das pacientes com miomatose uterina e o subtipo subseroso foi o mais frequente (35,5\%), seguido do intramural (34\%) (MONLEÓN $J$ et al., 2018).

Quanto ao tipo de tratamento, o tratamento cirúrgico foi o mais frequente e a histerectomia foi o procedimento de escolha. A histerectomia é o tratamento cirúrgico definitivo dos casos de SUA, é eficaz porque remove a causa/fonte do sangramento. Nos Estados Unidos (EUA) 600000 histerectomias são realizadas anualmente (MATTHEWS ML, 2015).

Miomas sintomáticos, como aqueles que causam anormalidades menstruais, anemia por deficiência de ferro ou sintomas importantes (por exemplo, pressão / dor pélvica, sintomas obstrutivos), a histerectomia é uma solução definitiva. No entanto, não é a solução para mulheres que desejam preservar a fertilidade e / ou seu útero (VILOS GA et al.,2015).

Dentre os tratamentos clínicos, o uso do análogo de GnRH foi o mais prevalente. Chama atenção a média do número de doses desta medicação nas pacientes que foram submetidas a tratamento cirúrgico e das que não foram submetidas a tratamento cirúrgico tenha sido superior a três doses, o que pode causar diversos efeitos colaterais a saúde dessas mulheres além do efeito rebote da medicação, visto que a mesma deve ser 
usada para melhora dos parâmetros hematológicos, melhora dos sintomas, diminuição dos tamanhos dos miomas para melhor facilidade operatória e diminuição do tempo cirúrgico (YELA DA e BENETTI-PINTO CL, 2018).

O diagnóstico precoce e o tratamento adequado são pilares do atendimento. Quanto mais precoce for o diagnóstico, maiores são as opções terapêuticas e maior a eficácia do tratamento. Em nosso estudo foi evidenciado que as pacientes que evoluíam há mais tempo com SUA referiram histórico de tratamento clínico, compreendendo tratamento hormonal e/ou não hormonal e posteriormente evoluíram para tratamento cirúrgico. Houve relevância estatística nos dados apresentados. Sabe-se que a falha no tratamento clínico é indicação de tratamento cirúrgico, porém o tratamento inadequado da condição de saúde, como uso prolongado de contraceptivos sem monitoramento, doses inadvertidas de análogos de GnRH agravam a condição de saúde da paciente e torna a opção de tratamento cirúrgico em alguns casos a única opção terapêutica.

Vale ressaltar que $37,3 \%$ das pacientes com SUA abandonaram o tratamento, o que pode-se justificar pelo sucesso no tratamento indicado por ausência de sinais e sintomas ou, por outro lado, à demora no acesso aos resultados de exames complementares para fins de diagnóstico e tratamento e também ao fato de alguns pacientes procurem agilizar o tratamento entram em filas de atendimento em outros serviços e acabam conseguindo sua cirurgia mais rápido, não retornando assim ao PAM.

Fatores fundamentais para o sucesso terapêutico incluem a discussão com a paciente em termos das opções existentes, suas preferências e tolerância a eventuais efeitos colaterais, presença de contraindicações, desejo de contracepção e sintomas associados como dismenorreia assim como a intensidade do sangramento (FILHO ALS et al., 2015).

A literatura aponta desigualdades no acesso aos serviços de saúde em relação às regiões de saúde do Brasil, em particular na região Norte, onde os serviços de média e alta complexidade estão localizados na capital do Amazonas, dificultando o acesso pelas populações do interior, ribeirinhas e áreas remotas (GARNELO L et al., 2017). O fato da distância em relação a Manaus, condições socioeconômicas muitas vezes precárias, dificuldades de marcação de exames, diminui a adesão ao tratamento, ampliam o tempo de retorno ao serviço, com isso levando atraso na terapêutica e até mesmo agravamento do quadro e até mesmo abandono o tratamento.

Com a média de tempo de aproximadamente 2 anos e 3 meses em que a paciente começou apresentar sintomatologia de SUA até a procura de atendimento no serviço de ginecologia PAM Codajás, percebemos que ao chegar no serviço primário não houve a princípio um direcionamento diagnóstico/terapêutico voltado para essa paciente de modo que intervisse na sua sintomatologia, o que nos leva a crer a carência de preparo da atenção primária para 0 atendimento às pacientes com SUA como os médicos de família são frequentemente o primeiro ponto de contato para pacientes com SUA, eles devem estar familiarizados com diagnósticos diferenciais e investigá-lo adequadamente.

\section{CONCLUSÃO}

Os dados apresentados permitiram concluir que: a prevalência de $22,1 \%$ de SUA foi maior que a encontrada nos países subdesenvolvidos e a prevalência global. A Leiomiomatose foi a causa mais comum de SUA entre as causas estruturais. A endometrial foi a causa mais comum de SUA entre as causas não estruturais. O tratamento em mais de $50 \%$ dos casos foi cirúrgico. Chamou atenção os casos de abandono do tratamento e o tempo de evolução da doença sem conduta ou acompanhamento ambulatorial, comprometendo a qualidade de vida e saúde das pacientes. Os resultados desse estudo inédito na região amazônica possibilitaram o conhecimento do perfil clínico e epidemiológico, identificação das causas, tratamentos instituídos a este grupo de mulheres, além de permitir a identificação de problemáticas a serem trabalhadas no que se refere ao manejo destas pacientes e que devem ser discutidas com a equipe de saúde que faz o primeiro atendimento a esta mulher e que conduz e trata as mesmas, visando uma abordagem integral, de qualidade, resolutiva e individualizada. 


\section{REFERÊNCIAS}

1. FILHO ALS, et al. Sangramento uterino anormal: proposta de abordagem do Grupo Heavy Menstrual Bleeding: Evidence-Based Learning for Best Practice (HELP). Femina, 2015; 43(4):162-166.

2. FILHO ALS et al. Tratamento do sangramento uterino anormal : uma análise sob a perspectiva dos custos no Sistema Único de Saúde e da medicina suplementar. Reprodução e Climatério, 2016; 31(1): 31-36.

3. GARNELO L et al. Regionalização em Saúde no Amazonas: avanços e desafios. Ciência \& Saúde Coletiva, 2017; 22(4):1225-1234.

4. HARLOW SD, CAMPBELL OMR. Epidemiology of menstrual disorders in developing countries : a systematic review. BJOG: an International Journal of Obstetrics and Gynaecology, 2004; 111(1): 6-16.

5. INSTITUTO BRASILEIRO DE GEOGRAFIA E ESTATÍSTICA. Indicadores Sociais Municipais: uma análise dos resultados do universo do Censo Demográfico 2010. Rio de Janeiro:IBGE, 2011; 149p.

6. MATTHEWS ML. Abnormal Uterine Bleeding in Reproductive-aged Women. Obstetrics and Gynecology Clinics of North America, 2015; (42)1:103-115.

7. MISHRA D, SULTAN S. FIGO' s PALM - COEIN Classification of Abnormal Uterine Bleeding: A Clinicohistopathological Correlation in Indian Setting. The Journal of Obstetrics and Gynecology of India, 2017; 67(2):119125.

8. MONLEÓN J. et al. Epidemiology of uterine myomas and clinical practice in Spain: An observational study. European Journal of Obstetrics \& Gynecology and Reproductive Biology, 2018; 226:59-65.

9. MONTEIRO IU. Guia Prático Manejo do Sangramento Uterino Anormal ( SUA ). São Paulo: Federação Brasileira das Associações de Ginecologia e Obstetrícia, 2015; 28p.

10. MUNRO MG et al. FIGO classification system (PALM-COEIN) for causes of abnormal uterine bleeding in nongravid women of reproductive age. International Journal of Gynecology and Obstetrics, 2011; 113: 3-13.

11. NATIONAL COLLABORATING CENTRE FOR WOMEN'S AND CHILDREN'S HEALTH. Heavy menstrual bleeding. London: Royal College of Obstetricians and Gynaecologists; 2007; 165p.

12. NATIONAL INSTITUTE FOR HEALTH AND CARE EXCELLENCE (NICE). Assessment and management of heavy menstrual bleeding. London: Prescriber; 2018; 36p.

13. RIZVI G et al. Histopathological correlation of adenomyosis and leiomyoma in hysterectomy specimens as the cause of abnormal uterine bleeding in women in different age groups in the Kumaon region: A retroprospective study. Journal of Mid-Life Health, 2013; 4(1):27-30.

14. SINGH, S et al. Abnormal uterine bleeding in pre-menopausal women. Journal of obstetrics and gynaecology Canada, 2013; 35:473-475.

15. SINGH S et al. Abnormal Uterine Bleeding in Pre-Menopausal Women. Journal of Obstetrics and Gynaecology Canada, 2018; 40(5): e391-e415.

16. SRIPRASERT I et al. Heavy menstrual bleeding diagnosis and medical management. Contraception and Reproductive Medicine, 2017; 2:20.

17. SUN Y et al. Prevalence of abnormal uterine bleeding according to new International Federation of Gynecology and Obstetrics classification in Chinese women of reproductive age: A cross-sectional study. Medicine, 2018; 97(31):e11457.

18. TAVARES NUL et al. Fatores associados à baixa adesão ao tratamento farmacológico de doenças crônicas no Brasil.Revista de Saúde Pública, 2016; 50(Suppl 2):1-11.

19. VIEIRA S. Bioestatística, Tópicos Avançados. $2^{\mathrm{a}}$ ed. Rio de Janeiro: Elsevier, 2004; $216 \mathrm{p}$.

20. VILOS GA et al. The Management of Uterine Leiomyomas. Journal of Obstetrics and Gynaecology Canada. Canada, 2015; 37(2):157-178.

21. YELA DA, BENETTI-PINTO CL. Sangramento uterino anormal - Protocolos Febrasco №42. São Paulo: Federação Brasileira das Associações de Ginecologia e Obstetrícia, 2018; 20p. 